\title{
DIE ANALYSE ENGLISCHER TEXTE AUS LINGUISTISCHER UND PRAGMATISCHER SICHT *
}

\section{Literaturuissenschaft und Linguistik}

1.1. Nachdem lange Zeit hindurch Literaturwissenschaftler und Linguisten in mehr oder weniger glücklicher Isolation nebeneinander her gearbeitet hatten, hat sich in jüngster Zeit das Verhältnis grundlegend geändert. Auch in unserem Fach hat sich die Überzeugung mehr und mehr durchgesetzt, daß beide - wenn auch mit verschiedenen Methoden und Zielen weithin mit dem gleichen Gegenstand beschäftigt sind, nämlich der englischen Sprache. Die Einbeziehung transphrastischer, pragmatischer und funktionaler Aspekte, besonders auch auf dem Gebiet der Textlinguistik, hat Linguisten und Literaturwissenschaftler einander wieder näher gebracht. Beide können sich, bei einer solchen Ausweitung des Untersuchungsgegenstandes der Linguistik, als Vertreter einer Textwissenschaft verstehen. So plädiert z.B. Plett (1975: 12) für eine Textwissenschaft mit zwei Teilkomponenten, einer nicht-ästhetischen, oder textuellen, und einer ästhetischen, oder literarischen, und spricht sogar von einem identischen Gegenstand der Linguistik und Literaturwissenschaft. Daß diese Auffassung nicht ganz unproblematisch ist, werden wir im folgenden sehen.

1.2. Eine Brücke zwischen Literaturwissenschaft und Lingui-

* Eine frühere Fassung wurde als Vortrag beim Anglistentag 1978 in Wuppertal gehalten. Erst danach ist mir die Erörterung indirekter Sprechakte in König (1977: 28-29) zur Kenntnis gekommen. Für Kommentare danke ich den Teilnehmern der Diskussion in Wuppertal, Prof. H.Käsmann, B.Brömser, J.Monaghan, sowie meinen Kollegen H. Gneuss und W. Weiß, wobei ich dem letzteren auch wertvolle Hinweise verdanke.

20 Anglia 98/3-4 
stik, die eigentlich nie ganz abgebrochen war, stellt die linguistische Stilistik dar (vgl. bes. Enkvist, 1973). Im Gegensatz zur Generativen Grammatik hatte sie nie aus den Augen verloren, daß die Sprache nicht uniform, homogen und unveränderlich ist, sondern daß es z.B. varieties of English gibt. Wie die Herkunft des vieldeutig verwendeten Begriffs register zeigt, ist diese Einsicht vor allem in der britischen Linguistik, die sich von Firth ableitet, deutlich gewesen. Der Terminus wird häufig synonym mit style verwendet, welches seinerseits u.a. als Wahlmöglichkeit, als Abweichung von Normen, als situationsbedingte Varianten usw. definiert worden ist.

1.3. Für eine umfassende Analyse und Erklärung der Interpretation von Texten ist jedoch die Stilistik in mehrfacher Hinsicht unzulänglich. Sie kann pragmatische Faktoren, wie Sprecher und Adressat, die z.B. in einem Drama besonders wichtig sind, nicht mit einbeziehen. Dabei muß vor allem beachtet werden, daß es im dramatischen Dialog nicht nur einen, sondern zwei verschiedene Sender gibt, nämlich den impliziten Autor und die Figur und ihre Rolle. Der Dialog hat außerdem zugleich auch zwei Adressaten, nämlich die Figur auf der Bühne und das Publikum. Dieses doppelte Kommunikationssystem ist für Verständnis und Erklärung dramatischer Texte unbedingt zu berücksichtigen. Jedoch nicht nur die Unterscheidung verschiedener Kommunikationskreise, sondern auch Sprechakt- und Handlungstheorie haben für das Drama zentrale Bedeutung. Stierle (1975: 8, 49-55) plädiert im Rahmen einer "systematischen Literaturwissenschaft" sogar dafür, narrative Texte, ja überhaupt jede Art von Text, als Handlung aufzufassen. Am Beispiel von King Henry IV zeigt Weiß (1978: 115), wie Shakespeare durch den Monolog, d.h. die Information des Publikums als alleiniger Adressat, die Sympathie dieser Adressaten lenken kann. Damit werden also nicht nur ,zwei Informationsebenen, die der Figuren und die des Publikums, etabliert", sondern der Autor steuert das Publikum, er handelt durch sprachliche und dramatische Akte.

Stilistische Methoden sind aber auch in anderen Fällen unzulänglich. Dies gilt besonders, wenn der Kommunikationsprozeß selbst zum Gegenstand gemacht wird, wie in Sternes Roman Tristram Shandy. Hier reicht auch die Unterscheidung 
von Sprachfunktionen, z.B. im Anschluß an Bühler, Jakobson oder Halliday, und die Isolierung verschiedener Kommunikationskreise zu einer adäquaten Erklärung nicht mehr aus. Nach meiner Auffassung (vgl. 5.) können hier u.a. die Sprechakttheorie, insbesondere die Untersuchung indirekter Sprechakte, sowie die von dem Sprachphilosophen Grice (1975) aufgestellten Prinzipien der kooperativen Konversation eine Erklärung liefern. Schließlich ist die Stilistik unzureichend, wenn es um die Erklärung deiktischer Phänomene geht, d.h. wenn es auf den Standpunkt des Senders oder Adressaten bei der Interpretation sprachlicher Äußerungen und der Analyse von schriftlichen Texten ankommt. Ich werde darauf in 2.2. und 5.3. näher eingehen.

\section{Pragmatische und funktionale Ansätze in der Linguistik}

2.1. Die linguistische Pragmatik ist in der Forschung auf verschiedene Art und Weise definiert worden, so z.B. als Lehre von der Zeichenverwendung, als Linguistik des Dialogs, oder auch direkt als identisch mit der Sprechhandlungstheorie. An der philosophischen Tradition des Begriffs Pragmatik orientierte Definitionen sehen sie als den Aspekt der Semiotik, der allgemeinen Zeichenlehre, der sich mit der Relation zwischen Zeichen und Zeichenbenutzern befaßt. Schließlich kann man Pragmatik auch als das Studium von sprachlichen Handlungen und deren Kontexten definieren. Nach meiner Auffassung ist die linguistische Pragmatik eine funktionale Sprachbetrachtung, die sich mit Sprechakten und deren sprachlichen und außersprachlichen Kontexten, einschließlich der Sprechsituation, befaßt.

2.2. In der linguistischen Forschung hängt die Definition der Funktionen von Sprache z.T. eng mit den jeweiligen Zeichentheorien zusammen. Das binäre, mentalistische Zeichenmodell Saussures - in dem Wörter als untrennbare und arbiträre Verbindung von Vorstellung und Lautbild aufgefaßt wurden schloß Sprecher und Hörer aus (auch wenn sich Saussure explizit mit dem Kommunikationsprozeß befaßte) und abstrahierte damit vom Zeichenbenutzer und den Funktionen sprachlicher Zeichen. Dieses binäre Modell wurde im semiotischen Dreieck 
von Ogden und Richards zu einem dreiseitigen Modell mit den Ecken symbol, thought und referent erweitert, welches aber nach wie vor Hörer und Sprecher ausschloß. Somit waren beide Modelle nicht-pragmatisch.

Erst Bühler (1965: 28-33) bezog in seinem Organon-Modell der Sprache Sprecher, Hörer und außersprachliche Referenten in die Theorie mit ein, und unterschied drei Hauptfunktionen der Sprache: Ausdruck, Darstellung und Appell. Das sprachliche Zeichen als Instrument ist Ausdruck des Senders (Sprecher im gesprochenen, Schreiber oder Autor im geschriebenen Medium), dient der Darstellung von Gegenständen und Sachverhalten (außersprachlicher Referenten) und hat drittens die Funktion des Appells an den Empfänger (Hörer oder Leser). Bühler (1965: 30f.) bestreitet die Dominanz der Darstellungsfunktion nicht, betont aber die Mischung von Funktionen in der konkreten Realisierung eines Zeichens. Bühlers Ansatz ist zugleich semiotisch und kommunikativ, d.h. an den Funktionen im Kommunikationsprozeß orientiert. Gleichzeitig interessiert ihn die konkrete Sprechsituation und damit Fragen der Deixis, weshalb auch ein wichtiges Kapitel seines Werkes vom "Zeigfeld" und den "Zeigwörtern" handelt. Der Ursprung des Orientierungssystems, die "Origo des Zeigfeldes", ist der Sprecher an einem bestimmten Ort zu einer bestimmten Zeit. Dieser Nullpunkt läßt sich repräsentieren durch "die Zeigwörter hier, jetzt und ich" (1965: 102).

Als Sprachpsychologe hat Bühler (1965: 52) aber auch bereits 1934, also lange vor Austin, den Handlungscharakter des Sprechens erkannt und betont: "Denn jedes konkrete Sprechen steht im Lebensverbande mit dem übrigen sinnvollen Verhalten eines Menschen; es steht unter Handlungen und ist selbst eine Handlung" (Sperrung von Bühler). Jakobson, als Vertreter der Prager Schule von Bühler beeinflußt, erweiterte 1960 dessen Modell und unterscheidet sechs verschiedene Sprachfunktionen (vgl. König, 1977: 2f.). Er ist vor allem an der poetischen Funktion der Sprache interessiert, die sich auf den Fokus oder die Einstellung auf die Form der Botschaft, d.h. die Art und Weise der Informationsvermittlung, bezieht. Als Differenzkriterium für literarische Texte wird diese Funktion wohl heute nicht mehr akzeptiert. Auch Jakobsons 
Definition der metasprachlichen Funktion (1960: 356) als Fokus auf dem code, der Sprache selbst, ist nicht unumstritten.

Halliday (1978: 50, 128f.) unterscheidet drei bzw. vier Makro-Funktionen, oder besser Meta-Funktionen der Sprache, die er auch "functional components" nennt: ideational (aufgespalten in experiential und logical), interpersonal und textual. Die erste bezieht sich auf die sprachliche Erfassung unserer Erfahrung und logischer Beziehungen und ist der Darstellungsfunktion Bühlers vergleichbar. Die zweite Funktion betrifft die Beziehung zwischen Sprecher und Hörer, die dritte die Beziehungen im Text. Halliday unterscheidet die drei Meta-Funktionen von den sieben sprachlichen Funktionen, die man beim Spracherwerb des Kindes trennen kann (1978: 19f., 55f.), den "developmental functions", bei welchen die informative Funktion ("I've got something to tell you") ganz im Hintergrund, die instrumentale ("I want") und die regulatorische ("Do as I tell you") - die Searles directives entspricht - ganz im Vordergrund steht.

2.3.1. Die Untersuchung verschiedener Sprachfunktionen hängt, wie wir eben sehen konnten, eng zusammen mit dem Objektbereich der sogenannten Sprechakttheorie. Diese wurde vor allem von Searle (1969), aufbauend auf den Einsichten des Sprachphilosophen Austin, entworfen und durch die Einbeziehung indirekter Sprechakte und eine neue Klassifizierung von sprachlichen Handlungen (Searle, 1976) in jüngster Zeit weiterentwickelt. In Abwandlung des Titels von Austins Buch läßt sich der Gegenstand der Sprechakttheorie vielleicht folgendermaßen charakterisieren: How to do things with utterances. Austin hatte noch gefragt, welche Handlungen man mit bestimmten Worten ausführen könne. Bei Searle sind daraus nicht Sätze, sondern Äußerungen in einem bestimmten situativen oder sprachlichen Kontext geworden. Die neuere Sprechaktklassifikation läßt sich mit folgenden Beispielen aus Searle (1976) illustrieren, wobei die illokutiven Akte einmal explizit, das andere Mal implizit ausgedrückt sind:

\footnotetext{
Representatives: I state that John hit Bill / He is a liar.

Directives: I order you to leave / Go out.

Commissives: I promise to pay the money / You'll get it.
} 
Expressives: I apologize for stepping on your toe / Congratulations.

Declaratives: I find you guilty as charged / I resign.

Hierbei ist zu beachten, daß die Beispiele für implizit ausgedrückte Akte im Kontext und Kontrast mit den expliziten Formulierungen zu sehen sind. Isoliert - bzw. in anderem Kontext - können sie selbstverständlich meist auch für ganz andere Arten sprachlicher Handlungen als die angegebenen verwendet werden. Das gleiche Verb kann in verschiedenen Sprechakten gebraucht werden, worauf Searle (1976: 22) ausdrücklich hinweist: I WARN you to stay away from my wife ist ein directive; I WARN you that the bull is about to charge ist nach Searle ein representative, könnte aber auch anders, z.B. zugleich als directive interpretiert werden.

2.3.2. Neben der Existenz normaler Sprechakte, für deren Klassifikation theoretisch auch andere Kriterien als die von Searle verwendeten benutzt werden könnten, hat Searle (1975) auch eine Klasse von indirekten Sprechakten aufgestellt ${ }^{1}$. Er weist darauf hin (1975: 59), daß die Äußerung $I$ want you to do it "is incidentally meant as a statement, but it is also meant primarily as a request". Die Feststellung fungiert also als Aufforderung oder Bitte, ohne die Form eines Imperativs zu haben. Ein anderes Beispiel hierfür wurde in der Forschung ausführlich diskutiert: it's cold in here, womit z.B. zum Schließen des Fensters oder der Tür aufgefordert werden kann. Auch die Aufforderung can you give me a hand with this wheel? hat nicht die syntaktische Form eines Imperativs, sondern einer Frage. Searle (1975: 60) betont, daß hier zwei Sprechakte gleichzeitig ausgeführt werden, gibt aber zu, "that some sentences seem almost to be conventionally used as indirect requests", wie z.B. can you reach the salt? Searle stellt in diesem Aufsatz außerdem auch eine Verbindung zwischen den Prinzipien von Grice und der weiter entwickelten Sprechakttheorie her, worauf wir noch zurückkommen werden (vgl. 4.3.).

1 Vgl. hierzu Lauerbach (1979: 159-169, 220-231) besonders für die Ableitung solcher Sprechakte und für die Verwendung von Konditionalsätzen mit if (wobei Höflichkeitskonventionen eine große Rolle spielen), sowie Coulthard (1977: 25-27, 45, 64-69) und König (1977: 28-39). 
Zunächst möchte ich jedoch auf die Einwände, die König (1977: $33 \mathrm{ff}$.) gegen diese Konzeption vorgebracht hat, eingehen. Sein Haupteinwand ist, daß viele Äußerungen durch formale Kennzeichnung, d.h. illokutive oder illokutionäre Indikatoren (Searle, 1969: 30f., 62f. illocutionary force indicator) klar in ihrer Bedeutung festgelegt seien. Solche Indikatoren sind z. B. tagKonstruktionen, mit denen sich König ausführlich beschäftigt. In vielen Fällen erfordere weiter die "wörtliche" Interpretation einen besonderen Kontext. Eine "Mischungshypothese", die für die Eigenschaften von Imperativsätzen, bei nicht-imperativischer Form, herangezogen werden könne, sei möglicherweise als Ergänzung zu Inferenzprozessen anzunehmen. So wird das Vorherrschen bestimmter syntaktischer und lexikalischer Formen in indirekten Sprechakten angeblich besser erklärt. Das Resultat dieser formalen Kennzeichnung stelle eine Art Idiom dar. Bei der Auffassung von Searle (1975), die bestimmte Inferenzprozesse notwendig macht, würde auch "ein besonderer Interpretationsaufwand erforderlich". Für diese schwierige und langwierige Interpretation gebe es aber keine Beweise. König (1977: 35) kommt daher zu dem Schluß, daß sehr häufig "wo man von indirekt ausgeführten Sprechakten spricht... die zum Verständnis notwendigen 'Inferenzprozesse' zumindest stark routinisiert und konventionalisiert [sind]".

Hierzu muß zunächst bemerkt werden, daß die angesprochene Idiomatisierung, sowie die dabei auftretende formale Kennzeichnung sowohl synchronisch wie diachronisch eine graduelle Erscheinung ist. Morgan (1978: 261) argumentiert, daß Ausdrücke wie can you pass the salt? zwar konventionell, aber nicht idiomatisch sind, und unterscheidet zwei Arten von Konventionen: "conventions of language" und "conventions of usage", letztere für die eben genannten Fälle. Auf das generelle Problem der Inferenzprozesse werden wir (vgl. 4.) noch näher eingehen. Eine grundsätzlich mögliche doppelte Interpretation ist aber zumindest in den nicht gerade seltenen Fällen von Mißverständnissen, auf die König (1977: 21, 35, 39) selbst hinweist, unbedingt erforderlich. Searle (1969: 70) gibt schon in seiner ersten Untersuchung von Sprechakten das Beispiel der Äußerung einer Ehefrau auf einer Party it's really quite late, mit welcher gleichzeitig verschiedene Sprechakte ausgeführt 
werden können. Coulthard $(1977: 20,40)$ weist darauf hin, daß in Grüßen, Witzen und Ähnlichem mehr als ein Sprechakt gleichzeitig ausgeführt wird, und daß damit die Mehrdeutigkeit von Äußerungen sogar systematisch ausgebeutet werden kann.

\section{Textbegriffe und Textdefinitionen}

3.1. In der Entwicklung der Textlinguistik lassen sich, grob gesehen, zwei Phasen unterscheiden, die mit verschiedenen Definitionen von 'Text' zusammenhängen: textimmanente, transphrastische in der frühen, und kommunikativ orientierte, handlungstheoretische Definitionen in der jüngeren Forschung. So sah man zunächst Texte als durch ununterbrochene pronominale Verkettung konstituiert, wobei Substitution und Pro-Formen eine große Rolle spielten. In dieser Phase wurde 'Text' auch als kohärente Folge von Sätzen definiert. Noch 1976 sprechen Halliday \& Hasan (1976: 23) davon, daß ein Text durch Kohäsion und Register zusammen wirkungsvoll definiert werde. Er muß in beider Hinsicht coherent sein. Dies ist jedoch nicht gleichzusetzen mit cohesive, da Halliday \& Hasan die verschiedenen Arten von cohesion als semantische Relation zwischen zwei Elementen auffassen. Im Gegensatz zu transphrastischen Definitionen gehen handlungstheoretische Ansätze im allgemeinen davon aus, daß der Text selbst ein sprachliches Zeichen oder Super-Zeichen ist. Charakteristisch hierfür ist die Text-Definition in Gülich \& Raible (1977: 22, 25, 47), die auf dem Modell der Kommunikation von Bühler aufbaut. Mein eigener Textbegriff schließt sich weitgehend an einen solchen Ansatz an. Ich betrachte Texte, sowohl im geschriebenen wie im gesprochenen Medium, als komplexe Zeichen, die in Relation zu einem Sender, einem Empfänger und einem außersprachlichen Referenten stehen. Gleichzeitig sind Texte als Kommunikationsakte sprachliche Handlungen, oder genauer gesagt, Folgen von Sprechakten, die auch geschrieben sein können. Somit sind z. B. auch Dialoge, und insbesondere Folgen von Fragen und Antworten, Texte und daher Gegenstand der Textlinguistik ${ }^{2}$.

- Vgl. hierzu Deimer (1975), Fries (1975) und für die Behandlung von Frage-Antwort-Sequenzen unter sprechakttheoretischem Aspekt in Shakespeares Othello Coulthard (1977: 171-180). 
3.2. In Halliday \& Hasan (1976: 8) wird der Begriff der Kohäsion unter anderem folgendermaßen definiert: "cohesion is a semantic relation between an element in the text and some other element that is crucial to the interpretation of it. This other element is also to be found in the text". Sie unterscheiden fünf Arten von cohesion im Englischen: reference, substitution, ellipsis, conjunction und lexical cohesion. Conjunction ist dabei nicht zu verwechseln mit dem homonymen traditionellen grammatischen Terminus, und entspricht weithin den in Meyer (1975) postulierten “Satzverknüpfungsrelationen”. Meyer (1975: 32), der versucht ein Interpretationsmodell für situationsunabhängige Texte aufzustellen, definiert die Satzverknüpfungsrelationen als "jene inhaltliche Beziehung zwischen zwei Sätzen, über die die Satzbedeutungen mit der Textbedeutung vermittelt sind, und die die Funktionen dieser Sätze im Text beschreibt". Er betont die grundsätzlich semantische Natur dieser Relation, sowie die Tatsache, daß sie keineswegs immer durch explizite grammatische Konnektoren formal ausgedrückt sein muß. In diesem Zusammenhang weist er mehrfach auf die in der klassischen Rhetorik verwendeten verschiedenen Arten von Asyndeta hin.

Eine sehr ähnliche Beobachtung ist in Leech \& Svartvik (1975: 160-162) bei der Behandlung von sogenannten "general purpose links" wie z.B. and zu finden. Sie stellen fest: "any positive link between two ideas can be expressed by and". Dies läßt sich besonders gut an den folgenden Beispielen aus Quirk \& Greenbaum (1973: 257) demonstrieren. Hier ist die semantische Beziehung zwischen den beiden koordinierten Sätzen keineswegs identisch, und kann durch die in der Klammer hinzugefügten Konnektoren explizit gemacht werden. In he heard an explosion $A N D$ he (therefore) phoned the police ist eine Kausalbeziehung, in Robert is secretive AND (in contrast) David is candid dagegen eine Kontrastbeziehung gegeben. Leech \& Svartvik weisen im Zusammenhang mit general purpose links darauf hin, daß Konjunktionen semantisch keineswegs eindeutig sind, und daß Satzverbindungen wie die folgenden, mit einem "missing link", ganz verschiedene semantische Beziehungen haben können: take this medicine: it'll make you feel better; he had to look for a job - he had run out of money. Sio 
stellen fest (1975: 162), daß in diesen Fällen: "the connection is implicit, and has to be inferred by the reader".

\section{Inferenzstrategien}

4.1. Wie bereits erwähnt, ist die Kohärenz von Texten - ob sie nun aus Äußerungen eines Sprechers oder Autors bestehen oder aus Dialogen - grundsätzlich eine semantische Erscheinung, die nicht explizit auf der sprachlichen Oberfläche ausgedrückt sein muß. Bei der Interpretation und Erklärung dieser semantischen Beziehungen spielen gewisse Inferenzstrategien des Hörers oder Lesers eine große Rolle, die aus den Prinzipien kooperativer Konversation von Grice abgeleitet werden können.

Ein erstes Beispiel sind die von Geis \& Zwicky beschriebenen invited inferences, die zunächst nicht mit semantischer Kontiguität oder Satzverknüpfungsrelationen in Verbindung zu stehen scheinen. In den folgenden Beispielen

(1) If you mow the lawn, I'll give you five dollars

(2) If you don't mow the lawn, I won't give you five dollars

(3) Martha observed the children at play AND she smiled with pleasure

wird nach Geis \& Zwicky der Satz (2) von Satz (1) logisch nicht impliziert. Dennoch lädt (1) dazu ein, oder suggeriert (2) ("invites or suggests"), eine Tatsache, die Geis \& Zwicky (1971: 562) durch eine "tendency of the human mind" erklären, eine Tendenz "to perfect conditionals to biconditionals". Eine andere Klasse von invited inferences sind Satzgefüge, in denen eine zeitliche Abfolge in eine Kausalbeziehung umgedeutet wird, wie in Satz (3). Eine solche suggerierte Kausalbeziehung, wie auch alle anderen invited inferences betrachten Geis \& Zwicky als eine Unterklasse der von Grice postulierten Implikaturen, mit denen wir uns nun beschäftigen werden.

4.2. Eine generelle Strategie zur Erklärung solcher Inferenzen hat der Sprachphilosoph Grice mit dem Begriff der Implikatur entwickelt, der auf den von ihm aufgestellten Prinzipien kooperativer Konversation aufbaut. Das folgende Beispiel (4) von Grice soll dies illustrieren: 
(4) A: How is $O$ getting on in his job?

B: Oh, quite well, I think, he likes his colleagues, and he hasn't been to prison yet.

Hierbei ist der Kontext im weiteren Sinne, d.h. sowohl die Sprechsituation als auch Annahmen und Vorwissen der beiden Sprecher, welche oft als Präsuppositionen bezeichnet werden, relevant. Die Äußerungen der Sprecher A und B im Beispiel (4) beziehen sich auf einen gemeinsamen Freund $C$, der jetzt in einer Bank arbeitet. Die Antwort von B auf die Frage von A impliziert oder suggeriert offensichtlich mehr als sie aussagt. Grice (1975: 43f.) führt dafür - zur Unterscheidung von den logischen Termini imply und implication - die Bezeichnungen implicate und implicature ein ${ }^{3}$. Die Implikatur des Beispiels (4) ist nach Grice (1975: 50), daß C möglicherweise unehrlich ist. Die Erklärung dieser Implikatur baut auf dem Prinzip der kooperativen Konversation, dem Cooperative Principle auf, welches Grice (1975: 45) folgendermaßen definiert: "Make your conversational contribution such as is required, at the stage at which it occurs, by the accepted purpose or direction of talk exchange in which you are engaged." Innerhalb dieses generellen Prinzips lassen sich vier Kategorien unterscheiden, aus denen eine Reihe von Konversationsmaximen abgeleitet werden, die in gekürzter Form in (5) dargestellt sind:

(5) A. QUANTITY (of information):

1. Make your contribution as informative as is required.

2. Do not make your contribution more informative than is required.

B. QUALITY (Try to make your contribution one that is true)

1. Do not say what you believe to be false.

2. Do not say that for which you lack adequate evidence.

C. RELATION: Be relevant.

D. MANNER (how what is said is to be said) (Be perspicuous):

1. Avoid obscurity of expression.

2. Avoid ambiguity.

3. Be brief (avoid unnecessary prolixity).

4. Be orderly.

\footnotetext{
3 Offenbar als Resultatnomen wird weiter implicatum geprägt, von Grice selbst und auch sonst in der Literatur aber praktisch nicht verwendet. Implicature und seine deutsche Entsprechung bezeichnen also in der Regel sowohl einen Vorgang oder eine Handlung als auch deren Resultat.
} 
Grice betont, daß nicht alle diese Maximen gleich wichtig sind, und daß möglicherweise noch weitere conversational maxims benötigt werden ${ }^{4}$. Nach Grice (1975: 47) sind diese Konversationsmaximen aber nicht gleichzusetzen mit anderen ästhetischen, sozialen oder moralischen Maximen, die ebenfalls normalerweise von den Teilnehmern an einem Gespräch beachtet werden, wie z.B. "Be polite" ${ }^{5}$. Nur die Konversationsmaximen und die daraus resultierenden Implikaturen haben aber, nach Grice, eine besondere Verbindung mit dem Zweck des Redens und des Wechselgesprächs. Grice $(1975 \cdot 47)$ betrachtet das Sprechen nur als einen Sonderfall zielgerichteten Handelns ("one of my avowed aims is to see talking as a special case or variety of purposive, indeed rational behavior"). Er gibt daher auch Beispiele für analoge, nicht-verbale Handlungen, die den vier Konversationskategorien entsprechen.

Die Konversationsmaximen sind sicherlich recht generell formuliert und überschneiden sich teilweise mit Ratschlägen in stilistischen und rhetorischen Handbüchern. Letzteres ist jedoch an sich kein Mangel, wenn man bedenkt, da $\beta$ in der Textlinguistik in jüngerer Zeit Kategorien der traditionellen Rhetorik wieder stärker berücksichtigt und integriert werden. Kritik an der Formulierung der Maximen ist aber nicht verwunderlich ${ }^{6}$. Dennoch ermöglicht gerade die allgemeine Fassung, im Zusammenhang mit der Relativierung innerhalb des Cooperative Principle ("the accepted purpose or direction of talk exchange"), eine Anwendung auf sehr unterschiedliche Texte. Ein genaues Maß für die jeweils notwendige, überflüssige, oder relevante Information kann es nicht geben. Wesentlich ist aber, $\mathrm{da} ß$ sich aus einer Verletzung der Maximen relativ zum anerkannten Konversationsziel oder spezifischen Text Implikaturen ergeben.

- Vgl. den Vorschlag in Searle (1975: 76) für die weitere Maxime speak idiomatically unless there is some special reason not to, der allerdings nicht ganz unproblematisch ist.

- Vgl. Lauerbach (1979: 155-159) und die zahlreichen in dieser Arbeit diskutierten Beispiele für indirekte Sprechakte mit if im Englischen, die auf Höflichkeitskonventionen beruhen.

- Vgl. hierzu und zu einem Versuch der Modifizierung der Maximen der Quantität Lauerbach (1979: 100-102). 
Die aufgestellten Konversationsmaximen können auf verschiedene Art und Weise verletzt werden. Jedoch nur eine Art der Verletzung, das flouting of a maxim, die eklatante, offensichtliche Nichtbeachtung, führt zur konversationellen Implikatur und stellt damit die Verbindung zwischen dem Cooperative Principle und den Konversationsmaximen her. Grice (1975: 49) formuliert dies folgendermaßen:

He may FLOUT a maxim; that is, he may BLATANTLY fail to fulfill it... This situation is one that characteristically gives rise to a conversational implicature; and when a conversational implicature is generated in this way, I shall say that a maxim is being EXPLOITED.

Grice $(1975: 45,50)$ unterscheidet zwischen konversationellen oder Konversations-Implikaturen und konventionellen Implikaturen. Die Konversations-Implikaturen stellen eine Unterklasse der nicht-konventionellen Implikaturen dar. Sie müssen im Prinzip ableitbar sein, wobei Grice die Formulierungen "work out", "calculate" und "derivation" gebraucht. Er betont ganz klar (1975: 50):

even if it can in fact be intuitively grasped, unless the intuition is replacable by an argument, the implicature... will not count as a CONVERSATIONAL implicature; it will be a CONVENTIONAL implicature.

Grice selbst gibt keine Beispiele für konventionelle Implikaturen. Aus Searle (1975: 65f.) stammen die folgenden Beispiele, die seiner Auffassung nach jedoch z.T. auch in einen "wörtlichen" Sinn, also nicht nur als Aufforderungen gebraucht werden können:

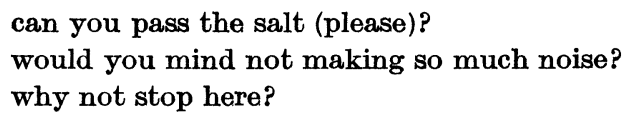

Grice (1975: $57 \mathrm{f}$.) nennt fünf Merkmale für KonversationsImplikaturen, weist aber darauf hin, daß trotz Berechnung oder Ableitung von Implikaturen eine Unbestimmtheit bleibe, da das Resultat der Implikatur in manchen Fällen eine Disjunktion verschiedener möglicher Erklärungen sein könne. In einem neueren Aufsatz greift Grice (1978: 114-116) die fünf Merkmale von Konversations-Implikaturen erneut auf und 
betont wieder, daß es keinen völlig schlüssigen Test dafür geben könne:

I very much doubt whether the features mentioned can be made to provide any such knock-down test, though I am sure that at least some of them are useful as providing a more or less strong prima facie case in favor of the presence of a conversational implicature (1978: 115).

Die folgenden Beispiele von Grice sollen das Funktionieren der Implikatur weiter illustrieren. Die Verletzung der 1. Maxime der Kategorie Quantity ${ }^{7}$ : wenn Grice, als Philosoph, auf die Bitte um ein Gutachten über einen früheren Studenten folgendes schreibt: "Dear Sir, Mr. X's command of English is excellent, and his attendance at tutorials has been regular. Yours sincerely". Die Implikatur, die sich hieraus ergibt, ist: der Bewerber ist in seiner Qualifikation als Philosoph nicht gerade überragend. Ironie und Metapher führt Grice (vgl. 1978: 123-125) auf eine offensichtliche Verletzung der 1. Maxime der Kategorie Qualität zurück. Die Äußerung $X$ is a fine friend muß in einer bestimmten Situation zur Implikatur des Gegenteils führen. Neben dem von Grice vorausgesetzten spezifischen Kontext sind bei diesem Beispiel jedoch auch Intonation oder Interpunktion (im geschriebenen Medium) relevant. In Grice (1978: 121f., 124) wird die Bedeutung von "stress" sowie eines "ironical tone" explizit angesprochen. Die Äußerung you are the cream in my coffee betrifft offensichtlich nicht den wahren Sachverhalt und muß daher metaphorisch interpretiert werden. Die Verletzung der Maxime der Relation wird durch einen Dialog in einer Party-Situation illustriert. Wenn der Sprecher A laut und deutlich die Äußerung Mrs. $X$ is an old bag von sich gibt und Sprecher B darauf the weather has been quite delightful this summer, hasn't it? äußert, so ist die daraus resultierende Implikatur der Tadel der Unhöflichkeit. Schließlich kann die offensichtliche Verletzung der Maxime Avoid obscurity of expression durch die Gegenwart eines Dritten, z.B. eines Kindes, das nicht verstehen soll, erklärt werden.

4.3. Eine Verbindung zwischen den Prinzipien von Grice und der weiter entwickelten Sprechakttheorie stellt Searle (1975)

7 Hier ließe sich zusätzlich auch eine Verletzung der Maxime der Relation annehmen. Vgl. Fußnote 10. 
in seinem Aufsatz über indirekte Sprechakte her. Sein folgendes Beispiel (1975: 61) kann dies illustrieren:

(6) A: Let's go to the movies tonight.

B: I have to study for an exam (vs. ...eat popcorn tonight).

Die Äußerung von B ist - in der Terminologie von Searle als sekundärer Sprechakt, d.h. in ihrer "wörtlichen" Bedeutung, eine Feststellung. Der primäre indirekte Sprechakt (primary illocutionary act), d.h. der Zweck der Äußerung selbst in einem bestimmten Kontext, ist die Zurückweisung eines Vorschlags ${ }^{8}$. Das Herausfinden dieses primären Sprechaktpotentials (primary illocution) durch den Hörer, d.h. seine Inferenzstrategien, interpretiert Searle als einen Vorgang in zehn Schritten, die in abgekürzter Form in (7) wiedergegeben sind.

(7) 1. I (A) have made a proposal to $B$, he (B) made a statement

2. I assume cooperative conversation ( $\rightarrow$ BE RELEVANT)

....

5. Therefore he probably means more than he says

....

8. Preparatory condition of accepting proposals is ability to DO it ....

10. Primary illocutionary point $=$ rejection of proposal.

Wichtig ist hierbei insbesondere der fünfte Schritt, die Erkenntnis, daß der Sprecher mehr sagen will als er oberflächlich ausdrückt. Der achte Schritt steht im Zusammenhang mit der gemeinsamen Hintergrundinformation von Sprecher und Hörer. Mit anderen Worten, beide wissen, daß die Vorbereitung auf Examen, aber auch ein Kinobesuch, relativ viel Zeit erfordert. Dies gilt nicht für andere Aktivitäten, weshalb z.B. eine Antwort von B wie: I have to eat popcorn tonight nicht als Zurückweisung des Vorschlags von A interpretiert werden könnte. Die Inferenzstrategien des Hörers oder auch Lesers, die Searle beschreibt, erfordern somit die Berücksichtigung der für Sprecher und Hörer gemeinsamen Hintergrundinformation, d. h. ihres Wissens von der Welt, sowie die Fähigkeit des Hörers

\footnotetext{
8 Searle (1975: 70) unterscheidet bei allen indirekten Sprechakten zwischen sentence meaning, der "wört)ichen" Bedeutung (literal illocution) und einer zusätzlichen speaker meaning, die der primary illocution entspricht.
} 
oder Lesers, Schlüsse zu ziehen. Sie involvieren weiterhin bestimmte Annahmen der Sprechakttheorie (so z. B. die preparatory condition im 8. Schritt) sowie die Prinzipien der kooperativen Konversation (im 2. Schritt). Illokutive Indikatoren, d. h. die formale Kennzeichnung der primary illocution durch lexikalische, syntaktische und andere Mittel (wie z. B. die Intonation) sind in diesen Fällen nicht vorhanden. Searle (1975: 68) weist selbst darauf hin, daß bei konventionalisierten direktiven Sprechakten häufig please als Indikator hinzugefügt werden kann. Wenn wir dies als Test-Möglichkeit benutzen, so erweist sich, daß can you reach the salt weniger stark konventionalisiert ist als can you pass the salt, please.

Das folgende weitere Beispiel für die Notwendigkeit von Inferenzstrategien wird in Coulthard (1977: 10) gegeben:

(8) A: Can you go to Edinburgh tomorrow?

B: B.E.A. pilots are on strike.

Er plädiert dafür, daß dieser Dialog - als Text - wenn auch nicht als cohesive, so doch als coherent betrachtet wird. Nur bei einer Antwort von B wie z. B. Yes, I can wäre der Text zugleich kohärent und kohäsiv. Die beiden Termini werden hier nicht genau wie bei Halliday \& Hasan verwendet (vgl. 3.1.), da Coulthard zwischen "grammatical cohesion" einerseits und "discourse coherence" andererseits unterscheidet und bei ihm zudem der Begriff des Registers in diesem Zusammenhang keine Rolle spielt. Über die notwendigen Inferenzschritte zur Interpretation der ersten Antwort ist an dieser Stelle bei Coulthard (1977) nichts ausgesagt.

\section{Textpragmatische Aspekte in "Tristram Shandy"}

5.1. Die Erklärungskraft indirekter Sprechakte und die Notwendigkeit des Einbezugs textpragmatischer Aspekte soll im folgenden an einigen Stellen aus Tristram Shandy illustriert werden. In seinem Aufsatz "Das gezielte Mißverständnis" spricht Berger (1972: 347, 340) davon, daß Sterne durch das Eingehen auf echte oder vorgetäuschte Mißverständnisse "eine ziemlich exakte Steuerung der Aufmerksamkeit seiner Leser" erreiche. Im Gegensatz zu dem Beispiel aus Shakespeares King Henry IV 
(vgl. 1.3.), wo die Sympathie, d.h. die emotionale Rezeptionshaltung des Adressaten gelenkt wurde, wird bei Sterne die Rezeptionsrichtung des Lesers indirekt gesteuert. Berger (1972: 340) macht darauf aufmerksam, daß der fiktive Leser ein fester Bestandteil des Romans ist und in vielfältiger Weise angesprochen wird, nämlich z.B. als Sir, Madam, Your Honour, my Lord, Your Reverences und Your Worships. Er stellt schließlich fest (1972: 341): "erst der scheinbare Versuch des Erzählers, ein mögliches Mißverständnis des fiktiven Lesers zu verhindern, macht den realen Leser deutlich auf die Ambiguität aufmerksam."

Über die von Berger gegebenen Beispiele hinaus sollen die folgenden Stellen diese Technik und andere Phänomene illustrieren und als indirekte Sprechakte auf der Grundlage der Verletzung von Konversationsmaximen erklären. Dabei kann keine vollständige Interpretation der Passagen gegeben werden. Diese sollen vielmehr zur Illustration verschiedener pragmatischer Ansätze und Aspekte von Texten dienen.

5.2.1. Im folgenden Zitat wird nach meiner Interpretation offensichtlich sowohl die 2. Maxime der Quantität als auch die 3. Maxime der Art und Weise verletzt.

Fair and softly, gentle reader! - - where is thy fancy carrying thee? - - If there is truth in man, by my great-grandfather's nose, I mean the external organ of smelling, or that part of man which stands prominent in his face -- and which painters say, in good jolly noses and well-proportioned faces, should comprehend a full third - - that is, measured downwards from the setting on of the hair. -Book III, ch. xxxiii (S. 160) ${ }^{9}$

Der Autor sagt mehr als erforderlich und auf eine umständliche Art und Weise. Die mehrfache und ausführliche Umschreibung von nose ist selbst in einem so wenig auf Kürze der Information bedachten Werk, also relativ zu diesem spezifischen Text, als Verletzung der entsprechenden Maximen aufzufassen. Ein Verstoß im Bereich der Kategorie Relation ist nicht so offensichtlich, da es für den Leser sehr schwierig ist festzustellen, was in Tristram Shandy relevant ist ${ }^{10}$. Aus der deutlichen Verletzung der Maximen ergibt sich die Implikatur, daß

\footnotetext{
- In der Klammer ist jeweils die Seitenzahl der von mir benutzten Ausgabe des Tristram Shandy in der Everyman's Library angegeben.

21 Anglia 98/3-4
} 
etwas anderes gemeint ist, als gesagt wird. Die Warnung vor einem Mißverständnis, d.h. einer anderen Interpretationsmöglichkeit, macht gerade auf diese aufmerksam. Bei den Inferenzschritten des Lesers spielt nun auch die Hintergrundinformation, d.h. das Wissen von der Welt eine Rolle, wodurch eine metonymische Beziehung zwischen den möglichen Referenten von nose hergestellt wird. Es handelt sich also um einen indirekten Sprechakt: gerade durch das Bestreiten der Ambiguität wird diese herbeigeführt und my great-grandfather's nose eine obszöne Bedeutung unterschoben.

5.2.2. Andere textpragmatische Aspekte bei der Analyse von Tristram Shandy müssen vor allem die Thematisierung des Kommunikationsprozesses selbst berücksichtigen. Die Verwendung graphischer Mittel, neben dem rein sprachlichen des Wortes, d.h. der Einbezug des gedruckten Mediums, spielt dabei eine große Rolle. Bei der Durchbrechung der Illusion durch Sichtbarmachen des Kommunikationsprozesses handelt es sich aber nicht um eine bloße Ausbeutung der von Jakobson und anderen in ähnlicher Weise postulierten metasprachlichen Funktion. Hier wird nicht bloß mit Sprache über das Sprachsystem, den sprachlichen Code, reflektiert, sondern wir haben es mit einem dynamischen Sprechen, einem sprachlichen und graphischen Handeln des Autors zu tun. Der Gegenstand ist der gesamte Kommunikationsproze $\beta$ mit all seinen verschiedenen Aspekten und Bedingungen, nicht einzelne Wörter.

5.2.3. Beim Sprechen mit dem fiktiven Leser über den Kommunikationsvorgang selbst kann sowohl der Produktions- als auch der Rezeptionsprozeß im Mittelpunkt stehen. Die Herstellung des von Adressaten aufgenommenen Textes wird im folgenden Zitat bewußtgemacht.

I will not finish that sentence till I have made an observation upon the strange state of affairs between the reader and myself, just as things stand at present... I am this month one whole year older than I was this time twelve-month; and having got, as you perceive,

10 Grice (1975: 46) macht darauf aufmerksam, daß man u. U. die 2. Maxime der Quantität nicht als Verstoß gegen das Cooperative Principle, sondern bloß als "waste of time" betrachten könnte; jedoch "its effect will be secured by a later maxim", nämlich BE RELEVANT. 
almost into the middle of my fourth volume - and no farther than to my first day's life - 'tis demonstrative that I have three hundred and sixty-four days more life to write just now, than when I first set out; so that instead of advancing, as a common writer, in my work with what $I$ have been doing at it - on the contrary, I am just thrown so many volumes back.

Book IV, ch. xiii (S. 207)

Durch das explizite Ansprechen der zeitlichen und situativen Bedingungen der Textproduktion wird indirekt die aufgebaute Erzählsituation zerstört und die Unzulänglichkeit des sprachlichen Kommunikationsvorgangs spielerisch betont. Dabei wird insbesondere der Faktor Zeit bei der Entstehung des Textes, der im traditionellen Roman unberücksichtigt bleibt, hervorgehoben.

5.2.4. Verschiedene Aspekte der Rezeption werden in den folgenden Beispielen angesprochen.

How could you, Madam, be so inattentive in reading the last chapter? I told you in it, That my mother was not a papist. - Papist! You told me no such thing, Sir. ... Then, Sir, I must have miss'd a page. - No, Madam, - you have not miss'd a word. - - Then I was asleep, Sir. My pride, Madam, cannot allow you that refuge. ... I do insist upon it, that you immediately turn back, that is, as soon as you get to the next full stop, and read the whole chapter over again.

Book I, ch. xx (S. 43)

Hier wird ein fiktiver Dialog geführt, wobei der direkt angesprochene Leser selbst zu Wort kommt, aber im argumentativen Dialog mit dem übermächtigen Autor unterliegt. Die angeblich mißglückte Kommunikation wird zum Anlaß genommen, den Lesevorgang selbst zu thematisieren ("immediately", "the next full stop") und dem Adressaten bewußt zu machen.

Sprachliche und graphische (nicht-sprachliche!!) Mittel werden von Sterne als handelndem Autor in der folgenden Passage ausgebeutet:

Read, read, read, read, my unlearned reader! ... for without much reading, by which your reverence knows I mean much knowledge, you will no more be able to penetrate the moral of the next marbled page (motly emblem of my work!) ...

Book III, ch. xxxvi (S. 163f.) 
Die zuletzt angesprochene marmorierte Buchseite ist natürlich gerade nicht durch Lesen zu entschlüsseln. Sie gehört zu den verschiedenen graphischen Mitteln, die Sterne neben dem geschriebenen Wort verwendet, wie z.B. auch die bekannte Zeigefingerhand alter Annoncen (S. 82, 146; II, xii; III, xx). Andere Mittel des gedruckten Mediums sind Gruppen von Sternchen, die die Stelle von Graphemkombinationen einnehmen. Auf ihre Funktion bei Laurence Sterne haben unter anderem Berger (1972: 345) und Plett (1975: 289) hingewiesen.

5.3.1. Auf die komplexen deiktischen Phänomene in Tristram Shandy, insbesondere die Zeitproblematik, kann ich hier nicht näher eingehen. Anhand des folgenden Beispiels soll nur auf die Problematik hingewiesen werden:

It is about an hour and half's tolerable good reading since my uncle Toby rung the bell, when Obadiah was ordered to saddle a horse, and go for Dr. Slop, the man-midwife; - so that no one can say, with reason, that I have not allowed Obadiah time enough, poetically speaking, and considering the emergency too, both to go and come... Book II, ch. viii (S. 75)

Hier werden die zeitlichen Ebenen der Rezeption, der Produktion und der fiktiven Handlung in der beschriebenen Geschichte miteinander verknüpft und vermischt. Gleichzeitig wird, durch die deiktischen Verben go und come im Bereich der Orts-Deixis der Standpunkt der handelnden Figuren Toby und Tristrams Vater eingenommen. Wir werden auf dieses Problem, mit dem sich Fillmore in verschiedenen Arbeiten intensiv beschäftigt hat, noch zurückkommen.

5.3.2. Bei der Untersuchung des Zeigfelds der Sprache betont Bühler (1965: 79), daß das "konkrete Sprechereignis" im Bereich der sprachlichen Deixis dynamisch ist: "es ist eine komplexe menschliche Handlung". Bei dieser Handlung "hat der Sender nicht nur wie der Wegweiser eine bestimmte Position im Gelände, sondern er spielt auch eine Rolle, die Rolle des Senders abgehoben von der Rolle des Empfängers" (79, Hervorhebung von Bühler). So verweist das Zeigwort ich auf den Sender, $d u$ dagegen auf den Empfänger des aktuellen Sprechereignisses. Bühler befaßt sich aber auch eingehend mit der Möglichkeit, den Schnittpunkt des Koordinatensystems, die Origo des Zeigfeldes in der Vorstellung zu verschieben und 
bezeichnet dies als "Deixis am Phantasma". Zur Illustration dieser Versetzung in der Vorstellung zieht er auch die "Fabel von Mohammed und dem Berg" heran (1965: 136). Ohne diese näher zu erläutern weist Bühler hierbei auf zwei verschiedene Möglichkeiten hin. Der erste Fall ist, daß "der Berg zu Mohammed kommt", d.h. das Vorgestellte an den Ort des Vorstellenden versetzt wird. Der zweite Fall liegt vor, wenn "Mohammed zum Berge geht", d.h. wenn man sich in der Vorstellung an einen bestimmten Ort hinversetzt. Er geht schließlich auch auf die Wirkung der Wahl verschiedener Zeigwörter in literarischen Texten ein und illustriert dies folgendermaßen (1965: 138):

Angenommen der Held wird nach Rom geschickt und der Autor steht vor der Wahl, ob er erzählend mit einem dort oder hier fortfahren soll. "Dort stapfte er den lieben langen Tag auf dem Forum herum, dort...". Es könnte ebenso gut hier heißen; was ist der Unterschied? Das hier impliziert eine Versetzung Mohammeds zum Berge...

Mit dem Phänomen der Deixis am Phantasma kann also die Versetzung des Standpunkts des Lesers durch den Autor erklärt werden.

5.3.3. Genau mit diesem Problem, den Inferenzen des Lesers über den Standpunkt des Beobachters, befaßt sich auch Fillmore (1976: 102f) am Beispiel des Eröffnungssatzes einer Kurzgeschichte von Hemingway ("The Killers"):

(10) The door of Henry's lunchroom opened and two men came in.

Vor allem aufgrund des Verbs come wird der Standpunkt des Beobachters festgelegt, aber die sprachliche Formulierung erlaubt auch andere Schlüsse über die Natur des Vorgangs, wie Fillmore erläutert. Insgesamt soll gezeigt werden (1976: 102):

The first sentence of the story provides a good example of how inferences about the observation point can be drawn from a sentence quite independently of what would ordinarily be considered part of the meaning of the sentence.

5.4. Berger (1972: 341) hat bereits darauf hingewiesen, daß "die Wahl einer Dialogsituation zwischen Erzähler und fiktivem Leser" in Tristram Shandy manche Vorteile für den Autor mit sich bringt. Sterne selbst betont, daß Schreiben nur eine andere Art der Konversation sei, und es dabei vor allem 
auch um das unausgedrückte Übermittelte, d.h. um indirekte Sprechakte geht.

Writing, when properly managed (as you may be sure I think mine is) is but a different name for conversation... The truest respect which you can pay to the reader's understanding is to halve this matter amicably, and leave him something to imagine, in his turn, as well as yourself.

For my own part, I am eternally paying him compliments of this kind, and do all that lies in my power to keep his imagination as busy as my own.

Book II, ch. xi (S. 79)

Dies geschieht besonders durch die Ausbeutung von Konversations-Implikaturen. Für ihr Verständnis können die Konversationsmaximen von Grice und die Theorie indirekter Sprechakte einen Beitrag liefern.

Alle angesprochenen Phänomene in Tristram Shandy - indirekte Sprechakte, fiktive Leseranreden und Dialoge mit dem Leser, die Bewußtmachung des Kommunikationsvorgangs und des Kommunikationsmediums - illustrieren jedoch meiner Ansicht nach, daß die pragmatischen Aspekte mancher Texte so offensichtlich und wesentlich sein können, daß hier nur linguistische Ansätze, die nicht vom Sender, Empfänger und Kontext abstrahieren, Erklärungshilfen liefern können.

\section{Konversationsanalyse und Textwissenschaft}

6.1. Für dialogische Textsorten - ob sie nun dramatisch sind oder nicht - scheinen besonders auch die Methoden und Ergebnisse der Conversational Analysis, wie sie im 4. Kapitel von Coulthard (1977) dargestellt werden, fruchtbar zu sein. Es handelt sich dabei einerseits um einen theoretischen Ansatz und praktische Untersuchungen des Soziolinguisten Labov, andererseits um die Forschungsergebnisse einer kleinen Gruppe von Soziologen wie Sacks, Schegloff und Jefferson.

Labov (vgl. Coulthard, 1977: 64-69) konzentriert sich auf Antworten und stellt eine Reihe von Interpretationsregeln auf, die erklären, weshalb eine bestimmte Äußerung als Antwort interpretiert werden kann. Das folgende Beispiel (11) erinnert sehr an bereits besprochene Fälle: 
(11) A: Are you going to work tomorrow? B: I'm on jury duty.

Die Interpretationsregel von Labov stimmt im Prinzip mit dem Verfahren der zehn Inferenzschritte von Searle überein, ist aber einfacher. Andere Regeln von Labov erklären, weshalb Äußerungen von A als "request for confirmation", "request for action" oder "indirect request" interpretiert werden, und enthalten alle den Passus: die Äußerung von A "is heard as...".

Bei den soziologischen Untersuchungen der KonversationsStruktur werden Einheiten wie utterance, turn und move verwendet. Diese empirischen Forschungen haben sehr interessante Einsichten ergeben, sind aber auch nicht unproblematisch (vgl. Coulthard, 1977: 91f). Die Hauptschwierigkeit dabei ist, daß die Beschreibungskategorien nicht sehr streng definiert sind, und die Forscher sich vor allem auf unter soziologischen Gesichtspunkten relevante Fragen konzentrieren.

6.2. Abschließend soll noch einmal die eingangs angesprochene Frage einer einheitlichen Textwissenschaft aufgegriffen werden. Linguisten wie Literaturwissenschaftler haben es gleichermaßen mit Texten zu tun. Kann man nun einfach innerhalb einer solchen übergreifenden Textwissenschaft zwischen literarischen und nicht-literarischen d.h. den sogenannten expositorischen Texten unterscheiden?

So wendet z.B. Coulthard (1977: 170-180), nach meiner Ansicht erfolgreich, Methoden der Konversations-Analyse auf Shakespeare's Othello an. Bei der Untersuchung von FrageAntwort-Sequenzen ergeben sich dabei interessante Einsichten. Es zeigt sich, daß insbesondere das Nicht-Beantworten von Fragen und die Ablehnung der diesen zugrundeliegenden Annahmen, in bestimmten Szenen eine ganz besondere Rolle spielen. Damit werden aber nicht nur von den betreffenden Figuren, besonders Iago, sprachliche Handlungen ausgeführt, sondern auch Shakespeare steuert die Reaktionen und die Aufmerksamkeit des Publikums. Coulthard (1977: 174, 180) weist aber wiederholt darauf hin, daß es sich hierbei nicht um echte Konversation, sondern um "simulated conversation", um "simulated spoken interaction" handelt. Er betont nachdrücklich (1977: 170): 
we must of course exercise due care when we apply techniques developed for the analysis of conversation to invented sequences created and shaped for an artistic purpose...

Er erläutert weiter, daß die Konversationsmaximen von Grice bei literarischer Konversation eine besondere Bedeutung haben. So gilt die Maxime BE RELEVANT nicht bloß für den im Gespräch behandelten Gegenstand, sondern auch für die Entwicklung des Themas und die Charakterisierung der Figuren. Auch die 2. Maxime der Quantität wird vom Standpunkt des fiktiven Gesprächs häufig verletzt. Was vom Standpunkt der Figuren als überflüssige Information erscheinen mag, kann dazu dienen, das Publikum indirekt zu informieren.

Es muß weiterhin darauf hingewiesen werden, daß gerade im Drama die Fabel oder Geschichte sowohl durch Sprechakte als auch durch physische Handlungen aufgebaut wird. In einem literarischen Text wird ganz allgemein die Sprechsituation selbst überhaupt erst durch den Text geschaffen. Somit ist eine einfache Gleichsetzung von fiktiven Texten und der natürlichen Sprachverwendung in einer gegebenen Situation gefährlich und unzulänglich.

Dennoch sollten wir nicht vergessen, daß die Analyse englischer Texte eine Aufgabe für beide, den Literaturwissenschaftler und den Linguisten ist. Ihre Methoden und Ziele sind zwar verschieden; dennoch ist ihr Gegenstand, die englische Sprache und ihre Verwendung, weithin identisch. Sicherlich ist heute die Einheit des Faches nicht mehr in einer Person des Forschenden und Lehrenden zu verkörpern. Dennoch sollten Linguisten und Literaturwissenschaftler nicht vergessen, daß sie im Grunde mit demselben Gegenstand zu tun haben, nämlich mit geschriebenen und gesprochenen englischen Texten.

\section{MÜNCHEN}

\section{LEONHARD LIPKA}

\section{Bibliographie}

Berger, D.A. (1972), "Das gezielte Mißverständnis. Kommunikationsprobleme in Laurence Sternes Tristram Shandy", Poetica 5, 327-347.

Bühler, K. (1965), Sprachtheorie. Die Darstellungsfunktion der Sprache, 2. Aufl., Stuttgart. 
Coulthard, M. (1977), An Introduction to Discourse Analysis, London. Deimer, G. (1975), Argumentative Dialoge. Ein Versuch zu ihrer sprachwissenschaftlichen Beschreibung (Linguistische Arbeiten 25), Tübingen.

Enkvist, N.E. (1973), Linguistic Stylistics, The Hague-Paris.

Fillmore, C.J. (1976), "Pragmatics and the Description of Discourse", in Pragmatik/Pragmatics II, ed. S.J.Schmidt, München, 83-104.

Fries, U. (1975), "Topics and Problems in 'Dialogue-Linguistics"', Studia Anglica Posnaniensia 7, 7-15.

Geis, M. L. \& Zwicky, A.M. (1971), “On Invited Inferences”, Linguistic Inquiry 2, 561-566.

Grice, H.P. (1975), "Logic and Conversation", in Syntax and Semantics. Volume 3: Speech Acts, ed. P.Cole \& J.L.Morgan, New York, 41-58.

Grice, H.P. (1978), "Further Notes on Logic and Conversation", in Syntax and Semantics. Volume 9: Pragmatics, ed. P.Cole, New York, 113-127.

Gülich, W. \& Raible, W. (1977), Linguistische Textmodelle, München.

Halliday, M.A. K. (1978), Language as Social Semiotic. The Social Interpretation of Language and Meaning, London.

Halliday, M.A.K. \& Hasan, R. (1976), Cohesion in English, London.

Jakobson, R. (1960), "Closing Statement: Linguistics and Poetics", in Style in Language, ed. T.A.Sebeok, Cambridge, Mass., 350-377.

König, E. (1977), Form und Funktion. Eine funktionale Betrachtung ausgewählter Bereiche des Englischen (Anglistische Arbeitshefte 13), Tübingen.

Leech, G. \& Svartvik, J. (1975), A Communicative Grammar of English, London.

Lauerbach, G. (1979), Form und Funktion englischer Konditionalsätze mit 'if'. Eine konversationslogische und sprechakttheoretische Analyse (Linguistische Arbeiten 72), Tübingen.

Meyer, P.G. (1975), Satzverknüpfungsrelationen. Ein Interpretationsmodell für situationsunabhängige Texte, Tübingen.

Morgan, J.L. (1978), "Two Types of Convention in Indirect Speech Acts", in Syntax and Semantics. Volume 9: Pragmatics, ed. P. Cole, New York, 261-280.

Plett, H.F. (1975), Textwissenschaft und Textanalyse, Heidelberg.

Quirk, R. \& Greenbaum, S. (1973), A University Grammar of English, London.

Searle, J.R. (1969), Speech Acts. An Essay in the Philosophy of Language, Cambridge.

Searle, J.R. (1975), "Indirect Speech Acts", in Syntax and Semantics. Volume 3: Speech Acts, ed. P.Cole \& J.L.Morgan, New York, 59-82.

Searle, J.R. (1976), "A Classification of Illocutionary Acts", Language in Society 5, 1-23.

Sterne, L. (1781; 1912), Tristram Shandy (Everyman's Library, No. 617), London-New York. 
Stierle, K. (1975), Text als Handlung. Perspektiven einer systematischen Literaturwissenschaft, München.

Weiß, W. (1978), "King Henry IV: Personenkonstellation und Sympathielenkung. Zum Rezeptionsproblem von Henry IV", in: Sympathielenkung in den Dramen Shakespeares, ed. W.Habicht \& I. Schabert, München, 112-120. 